\title{
2-氨基吡啶在构建五元、六元氮稠环合成中的应用
}

\author{
陈 淇 $a, b$ 陈思鸿 ${ }^{a}$ 吴汉清 ${ }^{a}$ 曾晓晴 $a$ \\ 陈伟清 $a$ 孙国星 $*, b$ 汪朝阳 $*, a$ \\ ( $a$ 华南师范大学化学学院 教育部环境理论化学重点实验室 广州市生物医药分析化学重点实验室 广州 510006) \\ ( $b$ 澳门大学应用物理及材料工程研究院 教育部联合重点实验室 澳门 999078)
}

\begin{abstract}
摘要 2 -氨基吡啶是一类重要的合成子, 具有独特的双亲核结构, 可与酮、醛、酸、多官能团酯类以及卤代芳烃等化合 物反应，以构建五元、六元含氮稠杂环. 近年来，基于 2 -氨基吡啶的成环反应倍受关注. 鉴于此，以底物种类为分类依 据, 重点综述了近 5 年来基于 2 -氨基吡啶构建咪唑并 $[1,2-a]$ 吡啶、吡啶并 $[1,2-a]$ 嘧啶合成方法的研究进展, 总结了其在 有机合成方法学、药物合成和荧光探针等领域的应用, 并展望了基于 2 -氨基吡啶的绿色化成环及其应用的未来发展趋 势.
\end{abstract}

关键词 2-氨基吡啶; 咪唑并 $[1,2-a]$ 吡啶; 吡啶并 $[1,2-a]$ 嘧啶; 氮稠环化合物; $\mathrm{C}-\mathrm{N}$ 键构建; 逆合成分析

\section{Application of 2-Aminopyridines in the Synthesis of Five- and Six-Membered Azaheterocycles}

\section{Chen, $\mathrm{Qi}^{a, b} \quad$ Chen, Sihong $^{a} \quad$ Wu, Hanqing ${ }^{a} \quad$ Zeng, Xiaoqing ${ }^{a}$}
Chen, Weiqing ${ }^{a}$
Sun, Guoxing ${ }^{*, b}$
Wang, Zhaoyang*,a

( ${ }^{a}$ Key Laboratory of Theoretical Chemistry of Environment, Ministry of Education, Guangzhou Key Laboratory of Analytical Chemistry for Biomedicine, School of Chemistry, South China Normal University, Guangzhou 510006)

( ${ }^{b}$ Joint Key Laboratory of the Ministry of Education, Institute of Applied Physics and Materials Engineering, University of Macau, Macau 999078)

\begin{abstract}
Aminopyridine is a significant synthetic synthon, with unique dual nucleophilic structure. It can react with ketones, aldehydes, acids, multifunctional esters, halogenated aromatics and other compounds to synthesize five- and six-member azaheterocycles. In recent years, the cyclization reactions based on 2-aminopyridines have been under the spotlight. According to the different types of substrates, the research progress on the synthesis of imidazo[1,2- $a]$ pyridines and pyrido[1,2- $a$ ]pyrimidines based on 2-aminopyridines in the past 5 years is reviewed, and its applications in organic synthesis methodology, drug synthesis and fluorescent probes are summarized. Furthermore, the development trend of green cyclization and its application based on 2-aminopyridines in the future are prospected.
\end{abstract}

Keywords 2-aminopyridine; imidazo[1,2-a]pyridine; pyrido[1,2-a]pyrimidine; azaheterocycle; $\mathrm{C}-\mathrm{N}$ bond formation; retrosynthetic analysis

吡啶环上氮原子具有较强的吸电子效应, 且吡啶 $\alpha$ 位基团具有特殊的作用, 使吡啶 $\alpha$ 位的衍生物作为合成 子或中间体在有机合成、药物合成中日益引人瞩目 ${ }^{[1-3]}$. 其中, 2-氨基吡啶作为最典型的一种, 因其独特的双亲 核结构特点为人们所重视 ${ }^{[4]}$, 特别是基于吡啶环上的氮 与氨基的成环反应倍受关注 ${ }^{[5-7]}$.
目前，随着研究的不断深入，基于 2-氨基吡啶构建 的各种成环化合物, 如咪唑并 $[1,2-a]$ 吡啶与吡啶并 $[1,2-a]$ 嘧啶, 不仅在材料合成 ${ }^{[8]}$ 、苂光分子 ${ }^{[9]}$ 和染料 ${ }^{[10]}$ 等领域发挥了重要的作用，而且应用于抗菌 ${ }^{[11]}$ 、抗

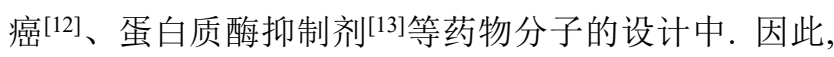
本文以不同底物种类为分类依据，重点综述了近 5 年来

\footnotetext{
* Corresponding authors. E-mail: wangzy@scnu.edu.cn; gxsun@um.edu.mo

Received April 5, 2021; revised April 29, 2021; published online June 1, 2021.

Project supported by the Guangdong Basic and Applied Basic Research Foundation (No. 2021A1515012342), and the Guangdong Provincial Science and Technology Project (No. 2017A010103016).

广东省基础与应用基础研究基金(No. 2021A1515012342)、广东省科技计划(No. 2017A010103016)资助项目.
} 
基于 2-氨基吡啶构建咪唑并 $[1,2-a$ 吡啶、吡啶并 $[1,2-a]$ 嘧啶的合成方法.

\section{1 构建咪唑环}

咪唑环作为咪唑并 $[1,2-a]$ 吡啶衍生物中的稠合单元 之一，可通过不同类型的合成方法和不同结构的底物构 建, 其中较为常见的是基于 2-氨基吡啶和羰基化合物的 反应(Scheme 1).

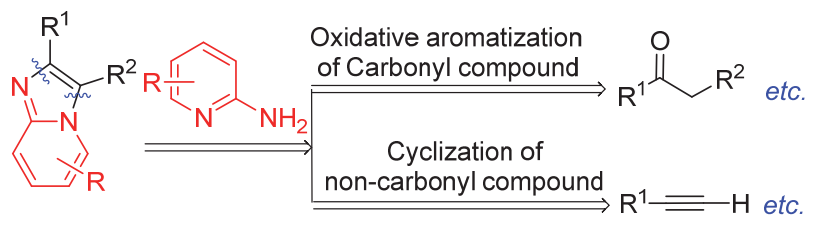

图式 12 -氨基吡啶合成咪唑并 $[1,2-a]$ 吡啶中咪唑环的逆合成 分析

Scheme 1 Retrosynthetic analysis for the synthesis of imidazo[1,2-a]pyridine from 2-aminopyridine

\section{1 以酮为原料}

$\alpha$-卤代苯乙酮衍生物是基于芳基乙酮类合成咪唑 并 $[1,2-a]$ 吡啶的关键中间体, 也可直接作为原料进行反 应. 例如, Berteina-Raboine 课题组 ${ }^{[14]}$ 以 $\alpha$-溴代苯乙酮和 2-氨基吡啶为原料, 采用可通过简单蒸馏而回收、循环 再用的桉油醇(Eucalyptol)作为溶剂, 在 $\mathrm{NaHCO}_{3}$ 存在下 得到 2-芳基咪唑并 $[1,2-a$ 吡啶 3. 该反应可能是由 2-氨 基吡啶 1 先与 $\alpha$-溴代苯乙酮 2 发生亲核取代反应, 互变 异构化后的中间体 $\mathbf{B}$ 分子内环化脱水得到产物(Scheme 2).

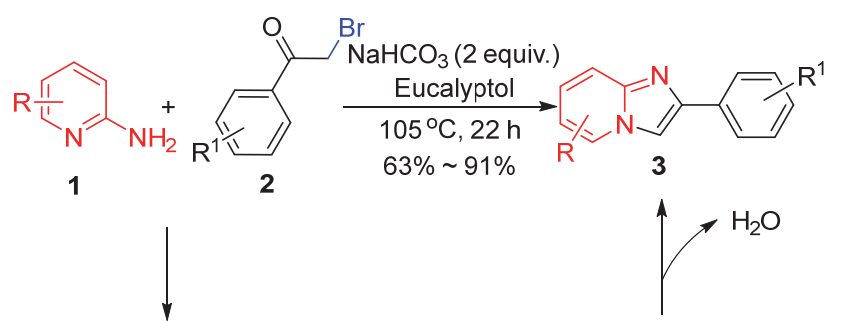

A<smiles></smiles>

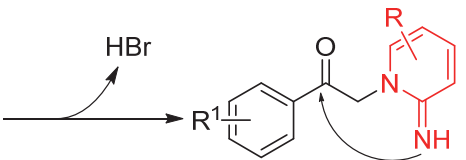

B
图式 2 2-氨基吡啶和 $\alpha$-溴代苯乙酮合成 2-芳基咪唑并 [1,2- $a$ ] 吡啶的反应机理

Scheme 2 Plausible mechanism for the synthesis of 2-arylimidazo[1,2- $a]$ pyridines from 2-aminopyridines and $\alpha$-bromoacetophenones

$\alpha$-溴代苯乙酮类化合物通常由苯乙酩类化合物的 $\alpha$-溴代反应制备, 且使用微波辐射的合成技术可以使 $\alpha$ -
溴代苯乙酮 2 与 2-氨基吡啶 1 在 $\mathrm{NaHCO}_{3}$ 存在下的缩合 反应在 $1 \mathrm{~min}$ 内完成(Scheme 3$)^{[15]}$.

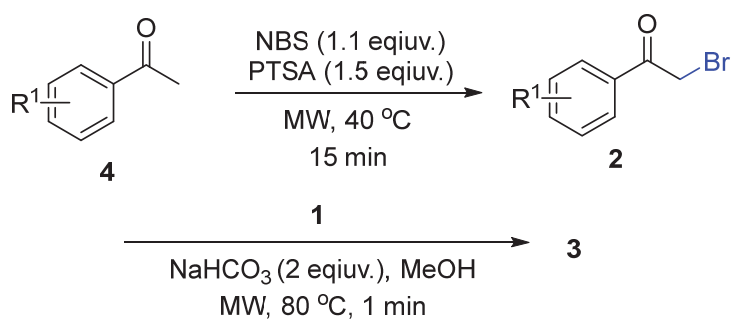

图式 3 微波辐射辅助 $\alpha$-溴代苯乙酮的合成及其与 2-氨基吡 啶的缩合反应

Scheme 3 Microwave radiation-assisted synthesis of $\alpha$-bromoacetophenone and its condensation reaction with 2 -aminopyridine

其实, 不需要碱, 直接添加氧化剂也能促使 $\alpha$-溴代 苯乙酮与 2-氨基吡啶反应，但得到的是溴代的咪唑并 $[1,2-a]$ 吡啶化合物. 例如, 徐盛涛课题组 ${ }^{[16]} \mathbf{1}$ 和 $\mathbf{2}$ 为 原料, 以叔丁基过氧化氢(TBHP)为氧化剂, 在 EtOAc 存 在下反应 $3 \mathrm{~h}$ 得到 3-溴代咪唑并 $[1,2-a$ ] 吡啶衍生物(Eq. 1). 反应中, $\alpha$-溴代苯乙酮 2 既充当底物又是卤源，提高 了原子经济性的同时使反应产物 5 具有良好的官能团耐 受性和可扩展性.

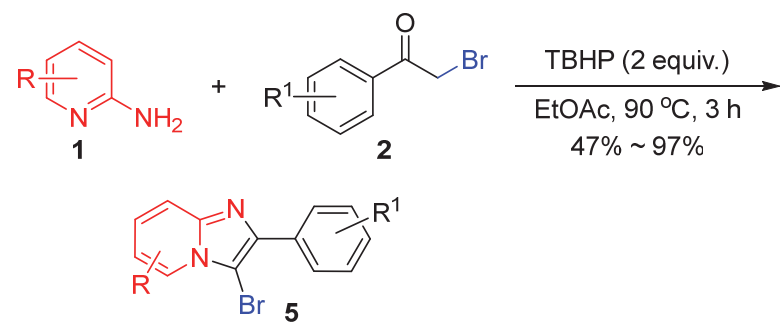

有机电化学合成能够避免使用有毒或危险的氧化 剂和还原剂 ${ }^{[17]}$, 亦可用于以 $\alpha$-溴代苯乙酮合成 3-溴咪唑 并 $\left[1,2-a\right.$ 吡啶类化合物. 例如, 黄精美课题组 ${ }^{[18]}$ 在电解 液为 $\mathrm{NH}_{4} \mathrm{Br}$ 的电池反应条件下, 利用一锅式电化学进行 合成, 产率为 $46 \% \sim 92 \%$.

若是以 ${ }^{n} \mathrm{Bu}_{4} \mathrm{NBF}_{4} / \mathrm{HI}$ 为电解液的电池反应条件, 还 能通过有机电化学合成法使芳基乙酮类化合物与 2-氨 基吡啶反应，合成咪唑并 $[1,2-a$ ]吡啶类化合物 3. 甚至 可以通过三组分反应, 在咪唑并 [1,2- $a$ ]吡啶的 3-位构建 $\mathrm{C}-\mathrm{S}$ 或 $\mathrm{C}-\mathrm{Se}$ 键 $\left(\right.$ Scheme 4) ${ }^{[19]}$.

其实, 更多情况下, 咪唑并 $[1,2-a$ 吡啶是由芳基乙 酮类化合物与 2-氨基吡啶在不同的卤代试剂和催化剂 共同作用下同时形成两个 $\mathrm{C}-\mathrm{N}$ 键而构建的, 并非绝对 需要底物中含有卤源. 一般而言， $I_{2}$ 常被用作该类反应 的卤源, 因为 $\mathrm{I}^{-}$在卤代烃进行亲核取代反应时易离去, 有利于加速反应进行 ${ }^{[20]}$.

例如，吴安心课题组 ${ }^{[21]}$ 以 2-氨基吡定 1 与苯乙酮 4 
为原料, 在 $\mathrm{I}_{2} / \mathrm{CuO}$ 的催化下合成了 2-芳基咪唑并 $[1,2-a]$ 吡啶, 产率为 $55 \% \sim 95 \%$, 其反应机理中涉及到 $\alpha$-碘代 苯乙酮的生成; 而赵培庆课题组 ${ }^{[22]}$ 开发了一种 $\mathrm{Cu}$ 基分 子篮掺杂的催化剂, 在 $I_{2}$ 存在下, 合成了 3 -碘代咪唑 $[1,2-a]$ 吡啶.

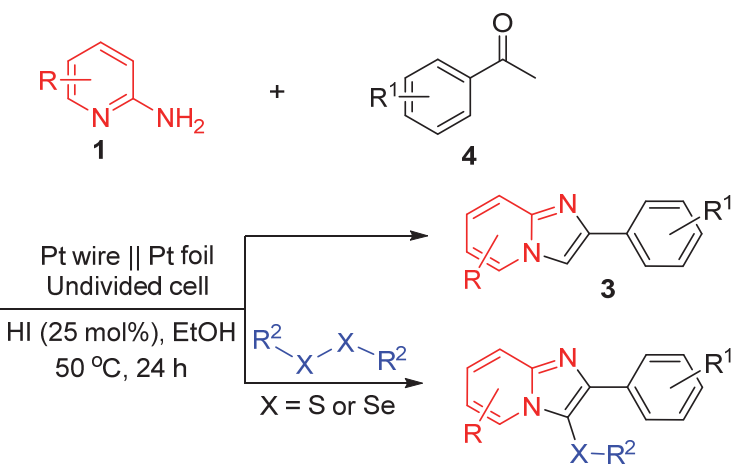

图式 4 通过有机电化学合成法由 2-氨基吡啶合成咪唑并 $[1,2-a]$ 吡啶类化合物

Scheme 4 Synthesis of imidazo[1,2-a]pyridines from 2-aminopyridine via organic electrochemical method

无金属参与的反应一直是化学工作者的研究热 点 ${ }^{[23]}$. 因此, Iida 课题组 ${ }^{[24]}$ 基于具有酶促特点的仿生催 化剂黄素(Flavin)开发了 $\mathrm{I}_{2} /$ Flavin 双催化体系, 在氧气氛 围中催化 1 与 4 的双组分反应, 得到 2-芳基咪唑并 [1,2-a]吡啶 3 (Eq. 2). 反应过程中, 黄素催化体系使生 成的 $\mathrm{I}^{-}$和 $\mathrm{H}^{+}$分别转化为 $\mathrm{I}_{2}$ 和反应唯一的副产物 $\mathrm{H}_{2} \mathrm{O}$, 促进 $I_{2}$ 循环使用的同时有利于减少环境污染. 值得注意 的是, 若是体系中还存在硫醇底物, 也可在咪唑并 [1,2$a$ ]吡啶的 3-位进一步构建 $\mathrm{C}-\mathrm{S}$ 键.

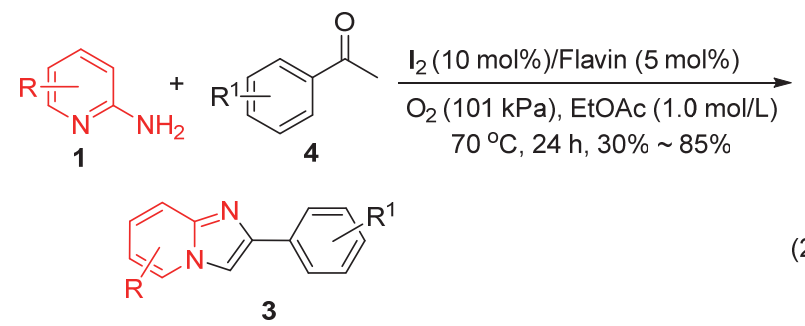

同样是在 1 与 $\mathbf{4}$ 的双组分反应中, 使用 $\mathrm{I}_{2} /$ 十二烷基 磺酸钠(SDS)胶束双催化体系时, 可得到 2-芳基咪唑并 [1,2- $a$ ] 吡啶, 并且依照此策略合成治疗胃溃疡药物 zolimidine, 能提高反应的原子利用率和产率 ${ }^{[25]}$. 若在 1、4 的反应体系中添加有机硫试剂磺酰肼 6 , 也可合成 咪唑并 $[1,2-a]$ 吡啶的硫醚化衍生物 7 (Eq. 3) ${ }^{[26]}$.

其实, 非芳香酮类化合物亦可与 2-氨基吡啶反应得 到咪唑并 $[1,2-a]$ 吡啶类化合物 ${ }^{[27]}$. 例如, 邓国军课题 组 ${ }^{[28]}$ 以环己酮类化合物 8 为原料进行反应, 在 $\mathrm{I}_{2} / \mathrm{O}_{2}$ 的条 件下制备得到了吡啶并 $[1,2-a]$ 苯并咪唑(Eq. 4). 值得注
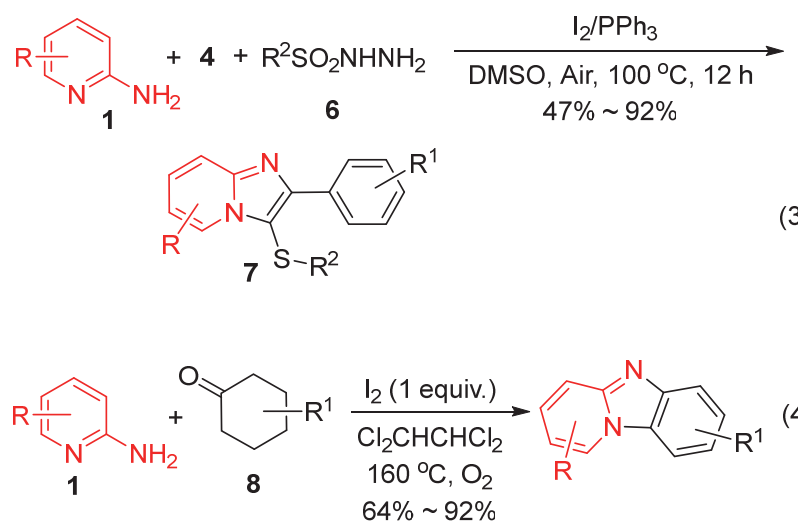

意的是, 在 $\mathrm{S}_{8}$ 的条件下, 环烷酮类与 2-氨基吡啶反应也 能得到咪唑并 $[1,2-a]$ 吡啶类化合物 ${ }^{[29]}$, 但生成的不是芳 构化产物(Eq. 5).

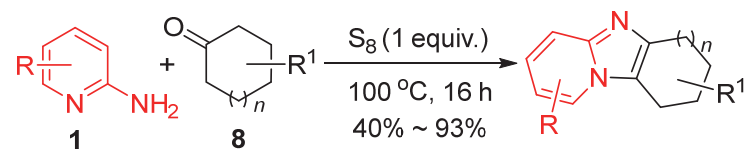

\section{2 以醛为原料}

事实上，并非只有酮羰基类化合物才可与 2-氨基吡 啶成环, 醛类也能与其发生成环反应 ${ }^{[30]}$. 例如, 苯乙醛 可与 2-氨基吡啶在 $S_{8}$ 的存在下发生双组分反应, 得到 3 -芳基咪唑并 $[1,2-a]$ 吡啶，反应的第一步是由 2-氨基吡 啶 1 与苯乙醛发生亲核加成反应(Eq. 6) ${ }^{[31]}$.

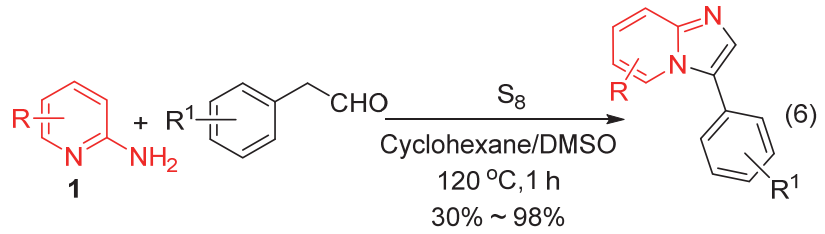

同样是 2-氨基吡啶与醛类的双组分反应，曹华课题 组 ${ }^{[32]}$ 则以 3-取代炔丙醛为原料, 在卡宾-金配合物的催 化下合成了 3 -酰基取代咪唑并 $[1,2-a]$ 吡啶. 机理研究表 明，产物中的酮羰基并非源自于原料中的醛羰基，而是 在反应中氧化得到的(Scheme 5a). 此后, 赣南师范大学 陈正旺课题组 ${ }^{[33]}$ 基于这一双组分反应体系，往其加入 $\mathrm{S}_{8}$ 作为底物, 在路易斯酸三氟甲基磺酸钪 $\left[\mathrm{Sc}(\mathrm{OTf})_{3}\right]$ 的 催化下得到了咪唑并 $[1,2-a$ ] 吡啶硫酮(Scheme $5 b$ ).

通常，通过一锅法多组分反应绿色高效地制备目标 分子是化学工作者的追求 ${ }^{[34]}$. 以 2-氨基吡啶、芳香醛和 末端炔烃为底物, 一锅法得到芳香酫中芳环位于咪唑环 的 2-位而非 3-位的产物 ${ }^{[35]}$. 例如, Rawat 课题组 ${ }^{[36]}$ 以 $\mathrm{CuIn}_{2}$ 掺杂的石墨烯量子点为催化剂, 三组分经历了生 成五元环的 5-exo-dig 分子内环化反应, 得到了 2,3-二芳 基取代咪唑并 $[1,2-a]$ 吡啶(Scheme 6). 


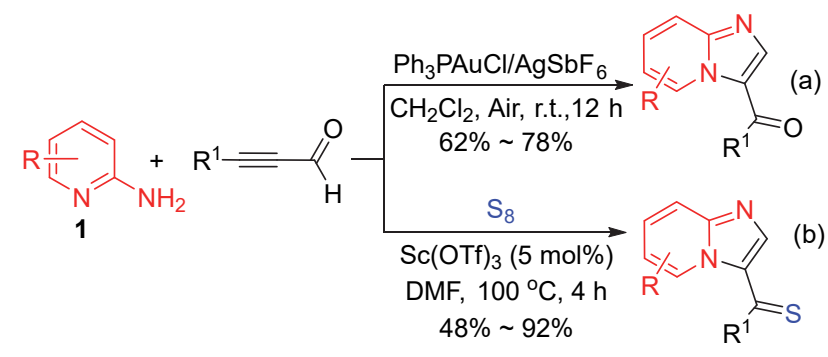

图式 52 -氨基吡啶与炔丙醛合成咪唑并 $[1,2-a]$ 吡啶类化合物 Scheme 5 Synthesis of imidazo[1,2-a]pyridines from 2-aminopyridines and propargyl aldehydes

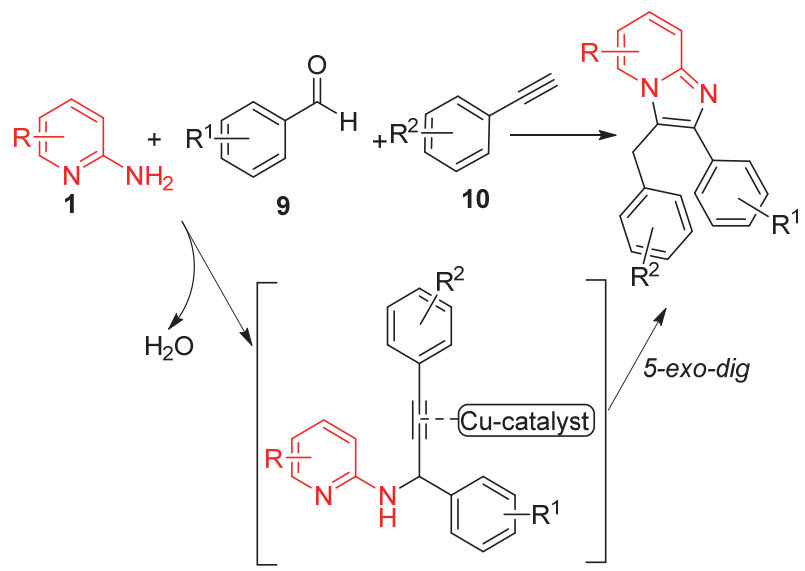

图式 62 -氨基吡啶、芳香醛和末端炔烃合成咪唑 $[1,2-a]$ 吡啶 反应的机理简图

Scheme 6 Simple scheme of the plausible mechanism for the synthesis of imidazo[1,2-a]pyridines from 2-aminopyridines, aromatic aldehydes and terminal alkynes

类似地，含参键的化合物如腈[37]或异腈 ${ }^{[38]}$, 亦能作 为 2-氨基吡啶 1、芳香醛 9 三组分反应体系的另一底物 发生成环反应. 例如, Thennarasu 课题组 ${ }^{[39]}$ 往 1、9 与异 腈的反应体系中加入催化剂 $\mathrm{I}_{2}$, 在室温下合成了具有荧 光探针特性的 2-芳基-3-仲胺咪唑并 [1,2- $a$ ] 吡啶(Eq. 7). 该系列探针能在水样或生物体内检测 $\mathrm{Zn}^{2+} 、 \mathrm{Fe}^{3+}$ 或 $\mathrm{Cr}^{3+}$ 而不受其他离子的干扰. 若是使用含 $\mathrm{Fe}_{3} \mathrm{O}_{4}$ 的纳米 颗粒为催化剂, 亦可高原子利用率地制得 2-芳基-3-仲 胺咪唑并 $[1,2-a]$ 吡啶 ${ }^{[40-41]}$, 产率均在 70\%以上.

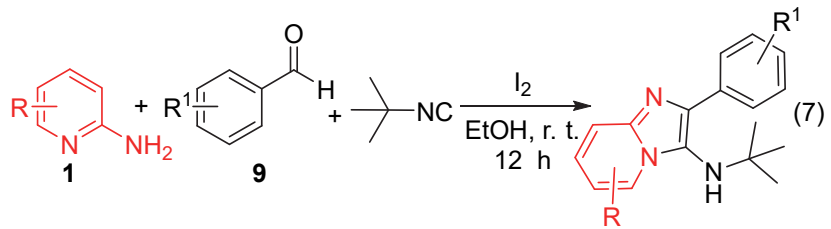

除了含碳碳参键、碳氮参键的化合物以外，硝基甲 烷亦能作为基于 2-氨基吡啶和芳香醛的三组分反应体 系的另一底物 ${ }^{[22]}$. 例如, 张占辉课题组 ${ }^{[43]}$ 使用磁性碳纳 米管负载的铜催化剂催化该一锅三组分反应, 合成了 3-
硝基-2-芳基咪唑并[1,2-a]吡啶(Eq. 8). 该反应在有氧条 件下进行，且催化剂能循环使用 8 次而不损失其活性.

$$
\text { (1) }
$$

\section{3 以其他羰基化合物为原料}

酸也是一类含有嵳基的化合物, 同样能与 2-氨基吡 啶发生成环反应 ${ }^{[44]}$, 特别是 $\alpha$-卤代羧酸 ${ }^{[45]}$. 例如, Sharma 课题组发现, 以氯乙酸为底物与 2 -氨基吡啶 1 反 应，经历亲核取代和分子内环化得到中间体 2-氯咪唑并 [1,2-a]吡啶 11. 该中间体可转化为用于分子自组装的氮 稠环化合物 12 和 $14^{[46]}$, 且后二者均可进一步将卤素硒 化得到具有抗菌活性的咪唑并 $[1,2-a]$ 吡啶化合物 13 和 $\mathbf{1 5}^{[45,47]}$ (Scheme 7).

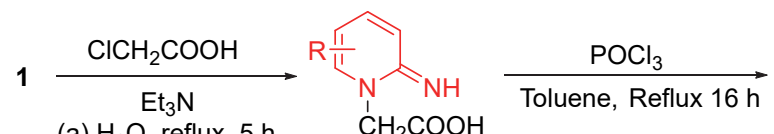

(a) $\mathrm{H}_{2} \mathrm{O}$, reflux, $5 \mathrm{~h} \quad \mathrm{CH}_{2} \mathrm{COOH}$

(b) $\mathrm{EtOH}, 5^{\circ} \mathrm{C}, 2 \mathrm{~h}$
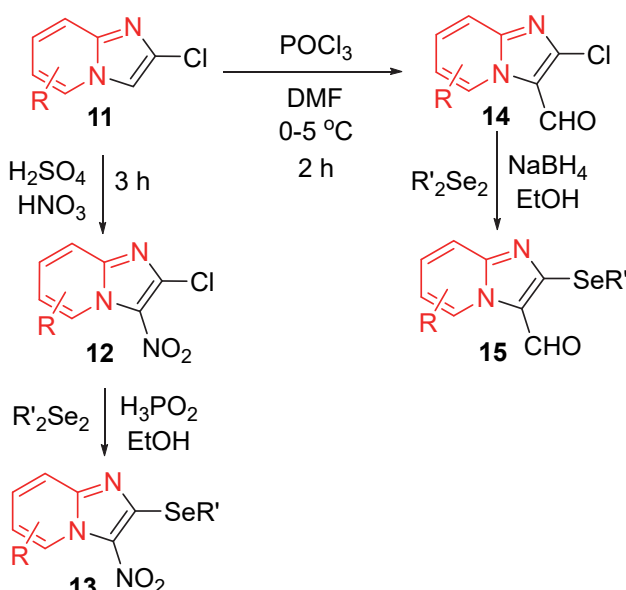

图式 72 -氨基吡啶和氯乙酸合成咪唑并 $[1,2-a]$ 吡啶类化合物 Scheme 7 Synthesis of imidazo[1,2-a]pyridine compounds from 2-aminopyridine and chloroacetic acid

除了酮、醛、羧酸外，一些二羰基化合物也与 2-氨

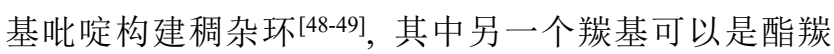
基 ${ }^{[50]}$. 例如, $\beta$-酮酸酯化合物能与 2-氨基吡啶在无过渡 金属催化条件下反应，得到 3-酯基取代咪唑并 $[1,2-a$ ]吡 啶 ${ }^{[51]}$ (Eq. 9).

\section{4 以芳基炔类、烯类等化合物为原料}

用于与 2-氨基吡啶进行成环反应的底物中, 芳基炔 


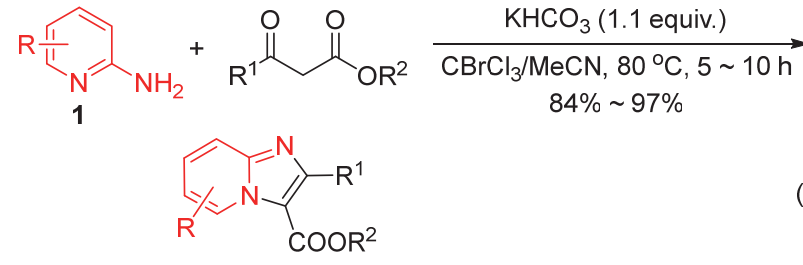

类化合物也较为常见 ${ }^{[52]}$. 此时, 通常需要金属催化 剂[53], 尤其是铜类催化剂 ${ }^{[54]}$. 其中, CuI 比较常见. 例如 1 与末端炔烃 10 在 $\mathrm{CuI}$ 和 $\mathrm{I}_{2}$ 的催化下, 经末端炔烃 $\alpha$ 位 卤代、氧化加成和还原消除历程, 合成 2-卤代-3-芳基取 代咪唑并 $[1,2-a]$ 吡啶 16 (Scheme 8, Method 1) ${ }^{[55]}$.

可以使用卤化铜 ${ }^{[56]}$ 和 $\mathrm{Cu}(\mathrm{OAc})_{2}{ }^{[57]}$ 等二价铜催化剂 进行催化. 以炔酸 ${ }^{[58]}$ 为底物, 在二价铜催化剂的作用下 得到卤代咪唑并 $[1,2-a]$ 吡啶 16 (Scheme 8, Method 2).

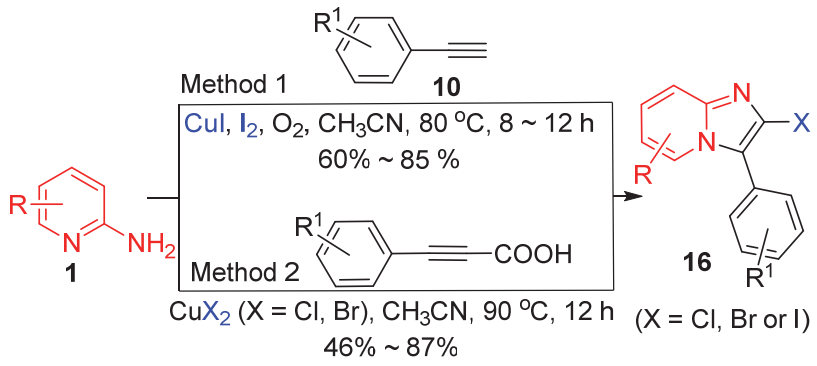

图式 8 2-氨基吡啶生成咪唑并 $[1,2-a]$ 吡啶的成环反应 Scheme 8 Cyclization reaction of imidazo[1,2-a]pyridines from 2-aminopyridines

若在 2-氨基吡啶与末端炔烃的反应体系中加入卤 代烃作为另一底物, 二价铜催化剂可在氧气氛围中催化 该三组分反应，得到 2-芳基-3-酰基取代咪唑并 $[1,2-a]$ 吡 啶 ${ }^{[59]}$ (Eq. 10).

除芳基炔类化合物外，芳基烯类化合物(如肉桂酸 [60]、查尔酮[1] ${ }^{[1}$ 亦能与 2-氨基吡啶发生成环反应，且经 常也需要金属催化 ${ }^{[62]}$, 特别是铜催化剂 ${ }^{[63]}$. 例如, 孙
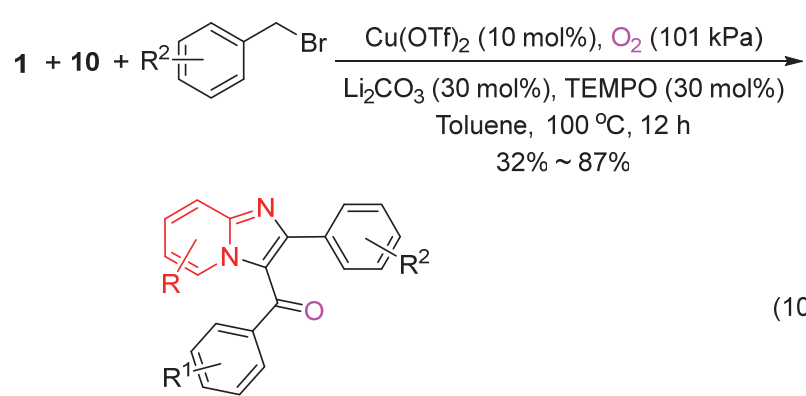

柏旺课题组 ${ }^{[64]}$ 发现, 肉桂醛与 2-氨基吡啶在 $\mathrm{CuI}$ 催化下, 经历迈克尔加成反应、脱氢氧化、质子转移、氧化加成 和还原消除历程，得到 2-芳基-3-甲酰基咪唑并[1,2- $a$ 吡 啶(Scheme 9).

不仅如此，一些胺类 ${ }^{[5]}$ 、芳香乙腈 ${ }^{66]}$ 、带有甲苯磺 酯基的缩酮类化合物 ${ }^{[67]}$ 等其他底物，都能在金属催化 下与 2-氨基吡啶反应成环. 例如, Guchhait 课题组 ${ }^{[68]}$ 最 近发现，易于获得的芳香嗍酸在 $\mathrm{Cu}(\mathrm{OAc})_{2}$ 与碱的存在 下，能与 2-氨基吡啶通过分子间 Chan-Lam $N$-芳基化和 分子内 $\mathrm{C}-\mathrm{H}$ 胺化反应, 生成吡啶并 $[1,2-a]$ 苯并咪唑 (Eq. 11).

\section{2 构建嘧啶环}

嘧啶环作为吡啶并 $[1,2-a]$ 密啶衍生物中的稠合单元 之一, 可由 2-氨基吡啶制备 ${ }^{[69]}$, 特别是通过其与多官能 团的酯类化合物等反应而构建(Scheme 10).

\section{1 以多官能团酯类为原料}

具有丙二酸酯骨架的多官能团化合物 ${ }^{[70-71]}$, 可与 2氨基吡啶进行环化反应，生成吡啶并 $[1,2-a]$ 嘧啶环类化 合物，最终应用于生物活性化合物的合成 ${ }^{[72]}$. 特别是最 常见的丙二酸二乙酯，因价格低廉，在含有吡啶并 $[1,2-$

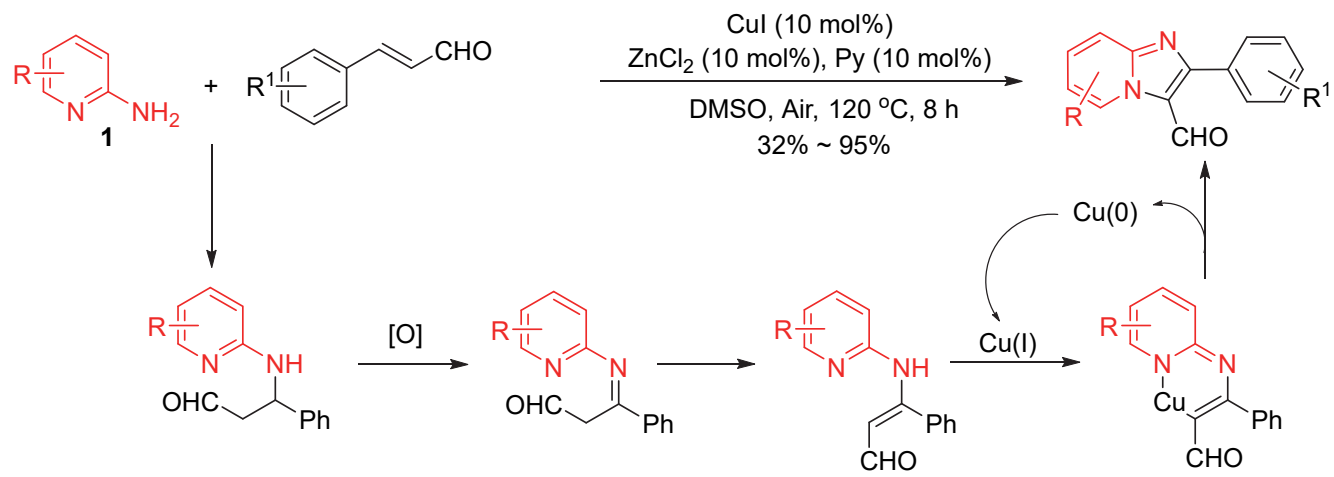

图式 92 -氨基吡啶和肉桂醛合成 2-芳基-3-甲酰基咪唑并 [1,2- $a$ 吡啶的反应机理

Scheme 9 Plausible mechanism for the synthesis of 2-aryl-3-formylimidazo[1,2-a]pyridines from 2-aminopyridines and cinnamaldehydes 

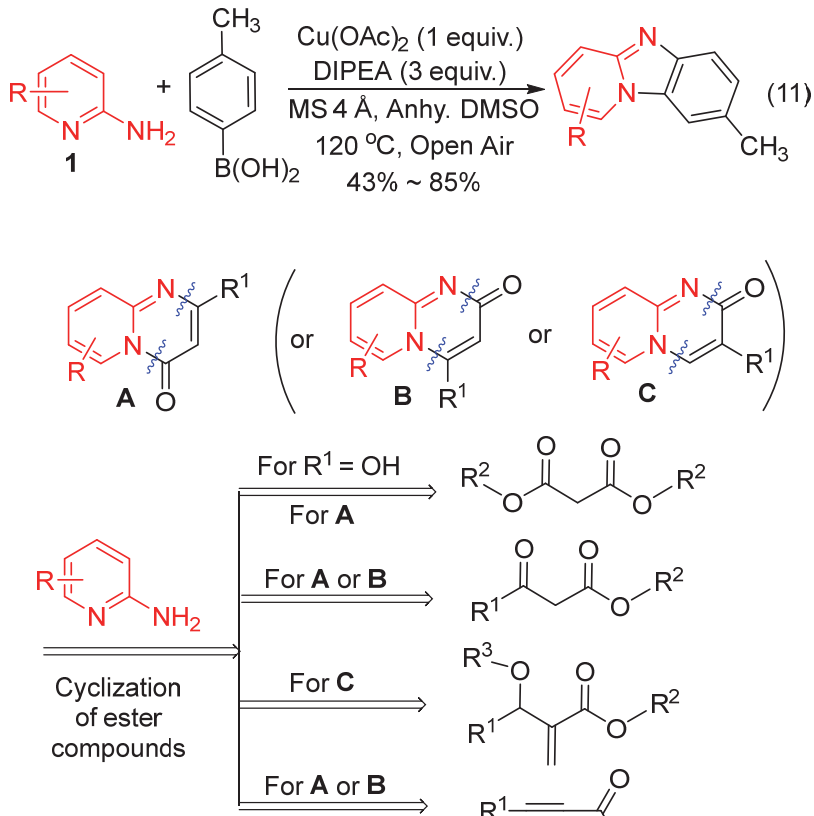

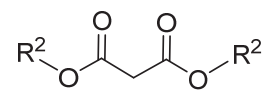<smiles>[R]OC(=O)CC([R])=O</smiles>

图式 10 2-氨基吡啶合成吡啶并 [1,2- $a$ ] 嘧啶中嘧啶环的逆合 成分析

Scheme 10 Retrosynthetic analysis for the synthesis of pyrido[1,2-a]pyrimidines from 2-aminopyridines

$a$ ]嘧啶稠杂环骨架的药物合成中备受关注 ${ }^{[73-74]}$. 例如, 杨财广课题组 ${ }^{[75]}$ 报道了丙二酸二乙酯与 2-氨基吡啶 1 在 无溶剂环境下的环化反应, 产物经多步反应, 可进一步 合成抗肾肿瘤的药物(Scheme 11).<smiles>CCOC(=O)CC(=O)OCC</smiles><smiles>Cc1cccnc1N</smiles><smiles>Cc1cccn2c(=O)c3cc(C(=O)NCCN4CCN(C)CC4)c(=N)n(CCc4cccs4)c3nc12</smiles>

图式 11 由 2-氨基吡啶类化合物和丙二酸二乙酯合成抗肾肿 瘤类药物

Scheme 11 Synthesis of anti-kidney tumor drugs from 2-aminopyridine type compound and diethyl malonate

若是以含更易离去基团的丙二酸二(2,4,6-三氯苯 基)酯为原料时, 成环时反应温度能够大幅度降低, 故 该策略在药物合成中也有所应用 ${ }^{[76-77]}$. 例如, Hwang 课 题组 ${ }^{[78]}$ 发现, 丙二酸二(2,4,6-三氯苯基)酯与 2 -氨基吡啶 1 的反应在吡啶边上引入嘧啶环骨架后, 产物经多步可
进一步合成丙肝病毒(HCV)抑制剂(Scheme 12).<smiles>Cc1ccc2nc(O)cc(=O)n2c1</smiles><smiles></smiles>

图式 12 由 2-氨基吡啶类化合物和丙二酸二(2,4,6-三氯苯基) 酯合成 HCV 抑制剂

Scheme 12 Synthesis of HCV inhibitor from 2-aminopyridine type compound and bis(2,4,6-trichlorophenyl) malonate

$\beta$-羰基酯类(如乙酰乙酸乙酯)也是基于 2 -氨基吡啶 构建吡啶并 [1,2- $a$ ]嘧啶化合物的常用原料 ${ }^{[79]}$. 例如, 刘 建辉课题组 ${ }^{[80]}$ 发现, 乙酰乙酸乙酯和 2-氨基吡啶在乙二 醇(EG)溶剂的存在下, 经迈克尔加成、质子转移、基团 离去与分子内环化的历程，得到 2-甲基-4H-吡啶并 [1,2- $a$ ]嘧啶-4-酮(Scheme 13).

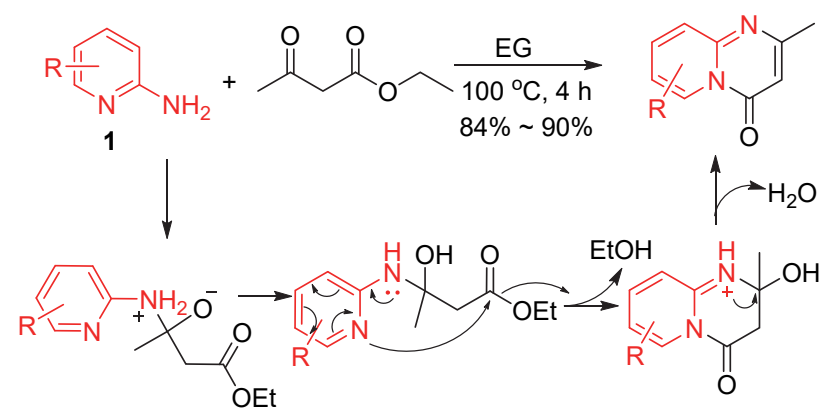

图式 132 -氨基吡啶和乙酰乙酸乙酯合成 $4 H$-吡啶并 $[1,2-a]$ 嘧啶-4-酮的反应机理

Scheme 13 Plausible mechanism for the synthesis of $4 \mathrm{H}$ pyrido[1,2-a]pyrimidin-4-ones from 2-aminopyridines and ethyl acetoacetates

值得注意的是，在该双组分反应体系中，不同的催 化剂可能会导致不同产物的生成: 以可循环使用的 $\mathrm{SiO}_{2}$ 负载对甲苯磺酸 $(\mathrm{TsOH})$ 为催化体系, 可得到 $4 H$-吡啶并 [1,2- $a$ 嘧啶-4-酮类化合物 ${ }^{[81]}$ (Eq. 12); 当反应体系中含 有 $\mathrm{CBrCl}_{3}$ 卤源时，反应生成咪唑并[1,2- $a$ ]吡啶(Eq. 9 $)^{[51]}$; 而 Maheswari 课题组 ${ }^{[82]}$ 发现，乙酰乙酸乙酯和 2-氨基吡 啶在单质硫的催化下，因硫与羰基之间的相互作用，得 到 4-取代-2H-吡啶并[1,2- $a$ ]嘧啶-2-酮(Scheme 14).

炔酸酯还能与 2-氨基吡啶进行成环反应，生成吡啶 并 $\left[1,2-a\right.$ 嘧啶类化合物 ${ }^{[80]}$, 产物中羰基的位置通常由反 应条件和底物决定 ${ }^{[83]}$. 例如, 芳基炔酸酯与 2-氨基吡啶 在三氟甲烷磺酸银的催化下反应, 得到 $4 H$-吡啶并

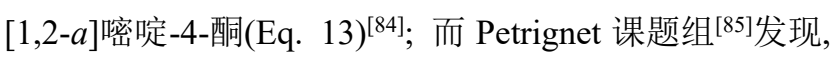




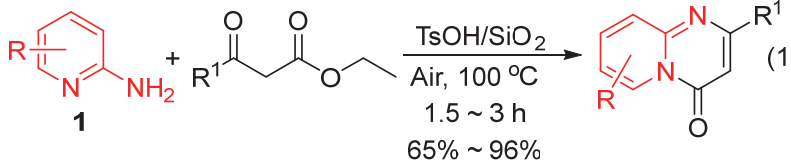

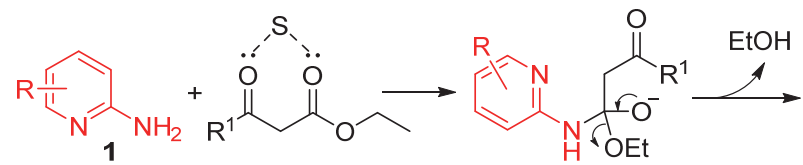

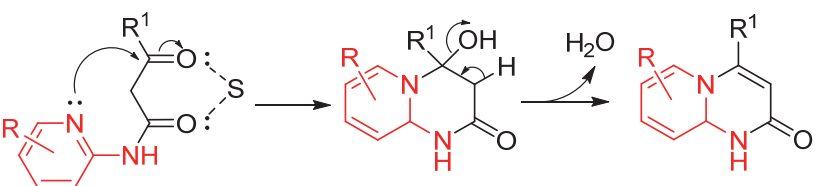<smiles>[R]c1cccc2nc(=O)cc([R])n12</smiles>

图式 142 -氨基吡啶和乙酰乙酸乙酯合成 $2 H$-吡啶并 $[1,2-a]$ 嘧啶-2-酮的反应机理

Scheme 14 Plausible mechanism for the synthesis of $2 H$-pyrido[1,2- $a]$ pyrimidin-2-ones from 2-aminopyridines and ethyl acetoacetates

若是以带有三氟甲基的炔酸酯为原料，因三氟甲基较强 的吸电子效应，使得氨基亲核进攻羰基，得到 4-三氟甲 基- $2 H$-吡啶并 $[1,2-a$ ]嘧啶-2-酮(Eq. 14).<smiles>[R]C[C@]1(C)C=Cc2nc(Br)cc(=O)n2C1[R]</smiles>

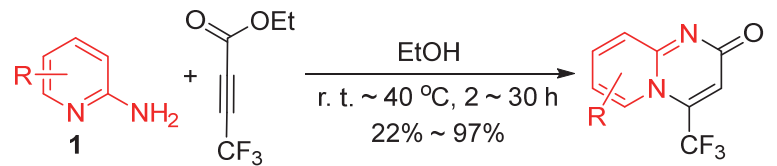

此外， $\alpha$-差圣甲基(或酯基保护的羟甲基)- $\alpha, \beta$-不饱和 酯亦能与 2-氨基吡啶发生反应, 生成吡啶并 $[1,2-a]$ 嘧 啶 ${ }^{[86-87]}$, 可由此在嘧啶环的 3-位引入取代基(Scheme $10)^{[88]}$. 例如, Alam 课题组 ${ }^{[89]}$ 发现, 在六氟异丙醇(HFIP) 的存在下, 二者经迈克尔加成、脱水、分子内酰基取代 和 $[1,3]$-氢迁移的历程, 可合成具有潜在生物活性的氮 稠环化合物(Scheme 15).

类似地, 含有多官能团的 $\alpha, \beta$-不饱和酰胺化合物 ${ }^{[90]}$ 也能与 2-氨基吡啶发生成环反应. 例如, 董德文课题 组 ${ }^{[91]}$ 报道了 3-取代-4H-吡啶并 $[1,2-a]$ 嘧啶-4-亚胺的合 成，第二步是中间产物在三氟甲磺酸酎 $\left(\mathrm{Tf}_{2} \mathrm{O}\right)$ 存在下的 分子内成环(Scheme 16).

基于 2-氨基吡啶构建吡啶并 $[1,2-a$ 嘧啶的多组分反 应, 也是化学工作者们的研究热点 ${ }^{[92-93]}$, 其中一个底物 可以是酯类化合物 ${ }^{[94]}$. 例如, Brahmachari 课题组 ${ }^{[95]}$ 发

现, 4-羟基香豆素与 2-氨基吡啶 1、芳香醛 9 在超声辐射 的条件下，通过一锅法策略进行反应，能得到 4-芳基取 代吡啶并 $[1,2-a]$ 嘧啶-4-酩(Eq. 15).

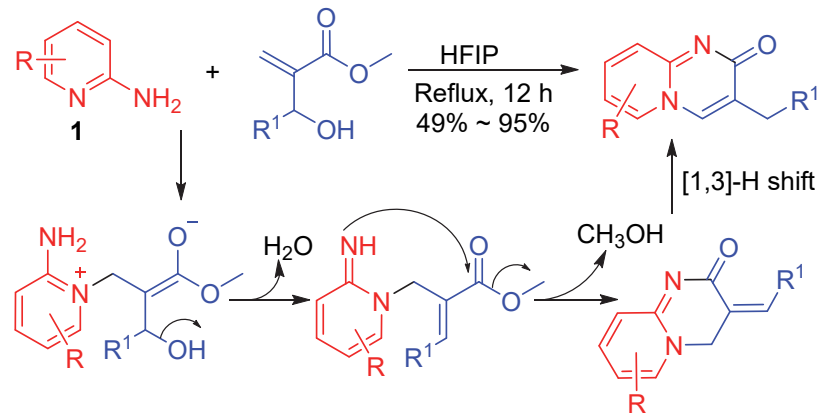

图式 152 -氨基吡啶和 $\alpha$-羟甲基烯酸酯合成 $2 H$-吡啶并 [1,2- $a$ ] 嘧啶-2-酮的反应机理

Scheme 15 Plausible mechanism for the synthesis of $2 \mathrm{H}$-pyrido[1,2- $a]$ pyrimidin-2-ones from 2-aminopyridines and $\alpha$-hydroxymethyl acrylates
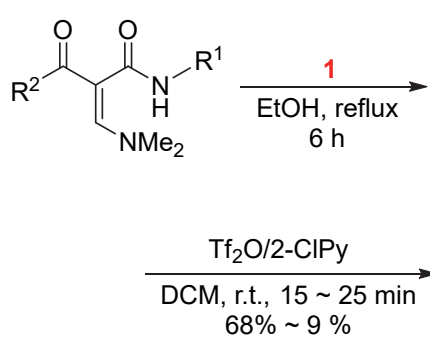<smiles>[R1]NC(=O)/C(=C\Nc1cc[R1]n1)C([R])=O</smiles><smiles>[R1]N=c1c(C([R])=O)cnc2c[R]ccn12</smiles>

图式 16 由酰胺与 2-氨基吡啶合成 3-酰基-4H-吡啶并 [1,2- $a$ ] 嘧啶-4-亚胺

Scheme 16 Synthesis of 3-acyl-4H-pyrido[1,2-a]pyrimidin4-imines from enaminones and 2-aminopyridines

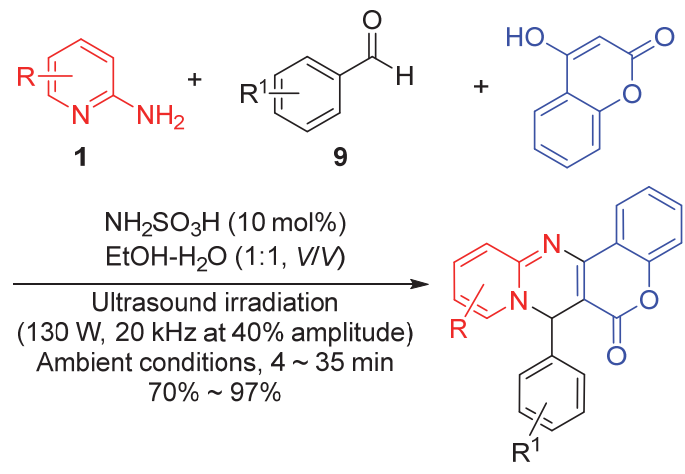

\section{2 以多官能团卤代芳烃类为原料}

除了多官能团的酯类外，多官能团的卤代芳烃类原 料也能与 2-氨基吡啶反应生成含吡啶并 $[1,2-a$ 嘧啶骨架 的三环化合物 ${ }^{[96]}$. 由于该类化合物不仅具有复杂天然 生物碱的骨架 ${ }^{[97]}$, 而且能应用为在红外和近红外区域 有苂光且荧光强度在弱酸环境下保持稳定的活细胞检 测的荧光染料 ${ }^{[98]}$ ，故其合成引人注目. 其中，基于邻卤 苯乙酮的合成最常见 ${ }^{[99]}$. 例如, Kaliappan 课题组 ${ }^{[100]}$ 最 
近报道, 邻溴苯乙酮与 2-氨基吡啶 1 反应, 经 UllmannGoldberg 型偶联反应、铜(I)催化氧化加成、还原消除、 分子内成环、甲基被氧化为 $\mathrm{CO}_{2}$ 离去的历程, 生成吡定 喹唑啉酮(Eq. 16).

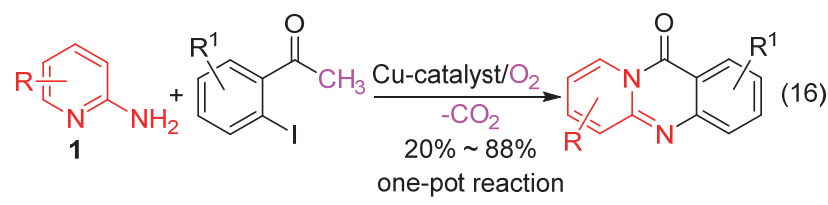

类似地, 2 -氟苯腈也可作为成环反应的底物, 在 ${ }^{t} \mathrm{BuOK}$ 的存在下生成 $6 H$-吡啶并 [1,2- $a$ ]喹唑啉-6-亚 胺 ${ }^{[101]}($ Scheme $17 \mathrm{a})$. 邻二卤芳烃也能与 2-氨基吡啶反应 成环, 但需要 $\mathrm{CO}$ 参与反应而构建羰基 ${ }^{[102]}$. 例如, 吴小 峰课题组 ${ }^{[103]}$ 报道, 以 1-氯-2-氟苯为底物, 在 $\operatorname{Pd}(\mathrm{II})$ 的催 化下亦能通过一锅法得到吡啶喹唑啉酮(Scheme 17b).

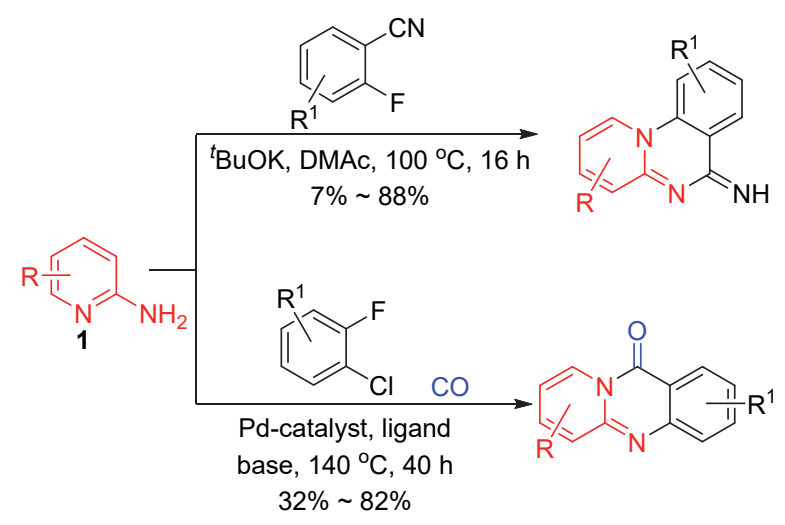

图式 172 -氨基吡啶生成吡啶并 $[1,2-a]$ 嘧啶类化合物的成环 反应

Scheme 17 Cyclization reaction of pyrido[1,2-a]pyrimidine compounds from 2-aminopyridines

\section{3 总结与展望}

综上所述，近年来基于 2 -氨基吡啶的成环反应取得 较大发展，基于酮、醛、酸、多官能团酯类以及卤代芳

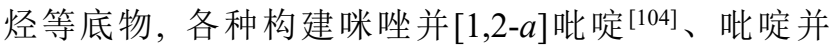
$[1,2-a]$ 嘧啶 ${ }^{[105]}$ 的合成方法已被报道. 这些方法不仅丰 富了有机合成方法学的研究途径, 而且在药物合成和苂 光探针 ${ }^{[106-107]}$ 等领域有较广泛的应用.

目前基于 2-氨基吡啶构建五、六元氮稠环的合成方 法多数局限于加热条件下的金属催化串联反应, 甚少利 用光催化合成、电化学合成等绿色合成方法. 可以预见, 随着人们对 2-氨基吡啶新反应研究的不断深入, 以及过 渡金属催化、氧化 $\mathrm{C}$ - $\mathrm{H}$ 反应与多组分反应等策略的发 展，开发更多反应底物与简单高效的含氮稠杂环合成方 法, 仍然值得关注. 特别是基于 2-氨基吡啶成环的绿色 合成发展(如光催化合成、电化学合成) ${ }^{[108]}$, 以及其在多
环药物活性分子的合成应用, 依然是未来重要的发展方 向.

\section{References}

[1] Kiefl, G. M.; Gulder, T. J. Am. Chem. Soc. 2020, 142, 20577.

[2] Cook, X. A. F.; de Gombert, A.; McKnight, J.; Pantaine, L. R. E.; Willis, M. C. Angew. Chem., Int. Ed. 2021, 60, 11068.

[3] Khatua, H.; Das, S. K.; Roy, S.; Chattopadhyay, B. Angew. Chem., Int. Ed. 2021, 60, 304.

[4] Ma, S. J.; Hill, C. K.; Olen, C. L.; Hartwig, J. F. J. Am. Chem. Soc. 2021, 143, 359.

[5] Bagdi, A. K.; Santra, S.; Monir, K.; Hajra, A. Chem. Commun. 2015, 51, 1555.

[6] Pericherla, K.; Kaswan, P.; Pandey, K.; Kumar, A. Synthesis 2015, $47,887$.

[7] Keiko, N. A.; Vchislo, N. V. Chem. Heterocycl. Compd. 2016, 52 , 222.

[8] Mutai, T.; Muramatsu, T.; Yoshikawa, I.; Houjou, H.; Ogura, M. Org. Lett. 2019, 21, 2143.

[9] Wu, Y. X.; Yuan, W.; Ji, H. Y.; Qin, Y. R.; Zhang, J.; Li, H. K.; Li, Y. H.; Wang, Y.; Sun, Y. F.; Liu, W. Dyes Pigm. 2017, 142, 330

[10] Yang, X.-Z.; Xu, B.; Shen, L.; Sun, R.; Xu, Y.-J.; Song, Y.-L.; Ge, J.-F. Anal. Chem. 2020, 92, 3517 .

[11] Thakur, A.; Pereira, G.; Patel, C.; Chauhan, V.; Dhaked, R. K; Sharma, A. J. Mol. Struct. 2020, 1206, 127686.

[12] Bin Sayeed, I.; Vishnuvardhan, M. V. P. S.; Nagarajan, A.; Kantevari, S.; Kamal, A. Bioorg. Chem. 2018, 80, 714.

[13] Lawson, M.; Rodrigo, J.; Baratte, B. Robert, T.; Delehouze, C.; Lozach, O.; Ruchaud, S.; Bach, S.; Brion, J. D.; Alami, M.; Hamze, A. Eur. J. Med. Chem. 2016, 123, 105.

[14] Campos, J. F.; Scherrmann, M. M. C.; Berteina-Raboine, S. Green Chem. 2019, 21, 1531 .

[15] Rodriguez, J. C.; Maldonado, R. A.; Ramirez-Garcia, G.; Cervantes, E. D.; de la Cruz, F. N. J. Heterocycl. Chem. 2020, 57, 2279.

[16] Liu, Y. P; Lu, L. X.; Zhou, H. P.; Xu, F. J.; Ma, C.; Huang, Z. J.; $\mathrm{Xu}$, J. Y.; Xu, S. T. RSC Adv. 2019, 9, 34671.

[17] Wang, B.-W.; Zhou, Y.-J.; Luo, S.-H.; Luo, X.-Y.; Chen, W.-Q.; Yang, S.-M.; Wang, Z.-Y. Chin. J. Org. Chem. 2021, 41, 171 (in Chinese).

(王柏文，周永军，罗时荷，罗晓燕，陈伟清，杨诗敏，汪朝阳， 有机化学, 2021, 41, 171.)

[18] Jian, W.-Q.; Wang, H.-B.; Du, K.-S.; Zhong, W.-Q.; Huang, J.-M. ChemElectroChem 2019, 6, 2733.

[19] Feng, M.-L.; Li, S.-Q.; He, H.-Z.; Xi, L.-Y.; Chen, S.-Y.; Yu, X.-Q. Green Chem. 2019, 21, 1619

[20] Yang, K.; Yao, C.; Gao, J.-J.; Chen, S.-H.; Zheng, X.-J.; Deng, L.-X.; Zheng, Y.-N.; Liu, M.-J.; Wang, Z.-Y. Chin. J. Org. Chem., 2020, 40, 4168 (in Chinese).

(杨凯，姚辰，高娟娟，陈思鸿，郑雪洁，邓璐璇，张毓娜，刘美 娟, 汪朝阳, 有机化学, 2020, 40, 4168.)

[21] Cai, Q; Liu, M. C.; Mao, B. M.; Xie, X.; Jia, F. C.; Zhu, Y. P.; Wu, A. X. Chin. Chem. Lett. 2015, 26, 881.

[22] Meng, X.; Yu, C. Y.; Chen, G. X.; Zhao, P. Q. Catal. Sci. Technol. 2015, 5, 372

[23] Wang, B.-W.; Jiang, K.; Li, J.-X.; Luo, S.-H.; Wang, Z.-Y.; Jiang, H.-F. Angew. Chem., Int. Ed. 2020, 59, 2338.

[24] Okai, H.; Tanimoto, K.; Ohkado, R.; Iida, H. Org. Lett. 2020, 22, 8002.

[25] Bhutia, Z. T.; Panjikar, P. C.; Iyer, S.; Chatterjee, A.; Banerjee, M. ACS Omega 2020, 5, 13333.

[26] Hu, S. P.; Du, S.Y.; Yao, Y. J.; Yang, Z.G.; Ren, H. J.; Chen, Z. K. Synlett 2019, 30, 625.

[27] Haouchine, A. L.; Kabri, Y.; Bakhta, S.; Curti, C. Nedjar-Kolli, B.; Vanelle, P. Synth. Commun. 2018, 48, 2159.

[28] Xie, Y. J.; Wu, J.; Che, X. Z.; Chen, Y.; Huang, H. W.; Deng, G.-J. Green Chem. 2016, 18, 667. 
[29] Nguyen, T. B.; Retailleau, P. Adv. Synth. Catal. 2017, 359, 3843.

[30] Frett, B.; McConnell, N.; Smith, C. C. Wang, Y. X.; Shah, N. P.; Li, H.-Y. Eur. J. Med. Chem. 2015, 94, 123.

[31] Tan, J.; Ni, P. H.; Huang, H. W.; Deng, G.-J. Org. Biomol. Chem. 2018, 16, 4227.

[32] Zhan, H. Y.; Zhao, L. M.; Liao, J. Q.; Li, N. Y.; Chen, Q. L.; Qiu, S. X.; Cao, H. Adv. Synth. Catal. 2015, 357, 46.

[33] Chen, Z. W.; Liang, P.; Xu, F.; Qiu, R. L.; Tan, Q.; Long, L. P.; Ye, M. J. Org. Chem. 2019, 84, 9369.

[34] Wu, H.-Q.; Yang, K.; Chen, X.-Y.; Arulkumar, M.; Wang, N.; Chen, S.-H.; Wang, Z.-Y. Green Chem. 2021, 21, 3782.

[35] Bhutia, Z. T.; Das, D.; Chatterjee, A.; Banerjee, M. ACS Omega 2019, 4, 4481.

[36] Purohit, G.; Kharkwal, A.; Rawat, D. S. ACS Sustainable Chem. Eng. 2020, 8, 5544.

[37] Rakhtshah, J.; Yaghoobi, F. Int. J. Biol. Macromol. 2019, 139, 904.

[38] Ghorbani-Choghamarani, A.; Taherinia, Z. J. Iran. Chem. Soc. 2020, 17, 59 .

[39] Mala, R.; Suman, K.; Nandhagopal, M.; Narayanasamy, M.; Thennarasu, S. Spectrochim. Acta, Part A 2019, 222, 117236.

[40] Soleimani, E.; Torkaman, S.; Sepahvand, H.; Ghorbani, S. Mol. Diversity 2019, 23, 739.

[41] Heydari, M.; Azizi, N.; Mirjafari, Z.; Hashemi, M. M. J. Iran. Chem. Soc. 2019, 16, 2357.

[42] Sun, J. W.; Yang, X. R.; Liu, Y.; Wang, Y.; Pan, Y. J. Heterocycl. Chem. 2020, 57, 1449.

[43] Zhang, M.; Lu, J.; Zhang, J.-N.; Zhang, Z.-H. Catal. Commun. 2016, 78, 26.

[44] Xiao, X. S.; Xie, Y.; Bai, S. Y.; Deng, Y. F.; Jiang, H. F.; Zeng, W. Org. Lett. 2015, 17, 3998.

[45] Kumar, S.; Sharma, N.; Maurya, I. K.; Verma, A.; Kumar, S.; Bhasin, K. K.; Sharma, R. K. New J. Chem. 2017, 41, 2919.

[46] Brar, S. K.; Kumar, S.; Pandey, S. K.; Bhasin, K. K.; Wangoo, N.; Sharma, R. K. J. Mol. Liq. 2018, 254, 208.

[47] Kumar, S.; Sharma, N.; Maurya, I. K.; Bhasin, A. K. K.; Wangoo, N.; Brandao, P.; Felix, V.; Bhasin, K. K.; Sharma, R. K. Eur. J. Med. Chem. 2016, 123, 916

[48] Sarlauskas, J.; Peciukaityte-Alksne, M.; Miseviciene, L.; Maroziene, A.; Polmickaite, E.; Staniulyte, Z.; Cenas, N.; Anusevicius, Z. Bioorg. Med. Chem. Lett. 2026, 26, 512.

[49] Karamthulla, S.; Khan, M. N.; Choudhury, L. H. RSC Adv. 2015, 5, 19724.

[50] Ramya, P. V. S.; Guntuku, L.; Angapelly, S.; Digwal, C. S.; Lakshmi, U. J.; Sigalapalli, D. K.; Babu, B. N.; Naidu, V. G. M.; Kamal, A. Eur. J. Med. Chem. 2018, 143, 216.

[51] Roslan, I. I.; Ng, K. H.; Chuah, G. K.; Jaenicke, S. Adv. Synth. Catal. 2016, 358, 364.

[52] Samanta, S. K.; Bera, M. K. Org. Biomol. Chem. 2018, 17, 6441.

[53] Rasheed, S.; Rao, D. N.; Das, P. Asian J. Org. Chem. 2016, 5, 1213.

[54] Cheng, C.; Ge, L.; Lu, X. H.; Huang, J. P.; Huang, H. C.; Chen, J.; Cao, W. G.; Wu, X. Y. Tetrahedron 2016, 72, 6866.

[55] Dheer, D.; Reddy, K. R.; Rath, S. K.; Sangwan, P. L.; Das, P.; Shankar, R. RSC Adv. 2016, 6, 38033.

[56] Liu, Y.; Zhang, Y. X.; Sun, J. W. J. Heterocycl. Chem. 2019, 56, 2804.

[57] Tran, R. Q; Jacoby, S. A.; Roberts, K. E.; Swann, W. A.; Harris, N. W.; Dinh, L. P.; Denison, E. L.; Yet, L. RSC Adv. 2019, 9, 17778.

[58] Liu, Y.; Wang, W. H.; Han, J. W.; Sun, J. W. Org. Biomol. Chem. 2017, 15, 9311.

[59] Li, X. W.; Wang, T. Z.; Lu, Y.-J.; Ji, S. M.; Huo, Y. P.; Liu, B. F. Org. Biomol. Chem. 2018, 16, 7143.

[60] Mutkule, N.; Bugad, N.; Mokale, S.; Choudhari, V.; Ubale, M. J. Heterocycl. Chem. 2020, 57, 3186.

[61] Meng, X.; Zhang, J. Q.; Chen, B. H.; Jing, Z. Q.; Zhao, P. Q. Catal. Sci. Technol. 2016, 6, 890 .

[62] Tzani, M. A.; Kallitsakis, M. G.; Symeonidis, T. S.; Lykakis, I. N. ACS Omega 2018, 3, 17947.

[63] Nguyen, O. T. K.; Ha, P. T.; Dang, H. V.; Vo, Y. H.; Nguyen, T. T.;
Le, N. T. H; Phan, N. T. S. RSC Adv. 2019, 9, 5501

[64] Zhai, L.-H.; Guo, L.-H.; Sun, B.-W. RSC Adv. 2015, 5, 93631.

[65] Rao, C. Q.; Mai, S. Y.; Song, Q. L. Org. Lett. 2017, 19, 4726.

[66] Tian, X. H.; Song, L. N.; Wang, M. M.; Lv, Z. G.; Wu, J.; Yu, W. Q.; Chang, J. B. Chem.-Eur. J. 2016, 22, 7617.

[67] McDonald, I. M.; Peese, K. M. Org. Lett. 2015, 17, 6002.

[68] Guchhait, S. K.; Saini, M. New J. Chem. 2020, 44, 308.

[69] Verma, K.; Tailor, Y. K.; Khandelwal, S.; Agarwal, M.; Rushell, E.; Kumari, Y.; Awasthi, K.; Kumar, M. RSC Adv. 2018, 8, 30430.

[70] Selvi, S.; Srinivasan, K. Eur. J. Org. Chem., 2017, 2017, 5644.

[71] Tsoung, J.; Bogdan, A. R.; Kantor, S.; Wang, Y.; Charaschanya, M.; Djuric, S. W. J. Org. Chem. 2017, 82, 1073.

[72] Liu, D. Y.; Zhang, J.; Zhao, L.; He, W. J.; Liu, Z. J.; Gan, X. H.; Song, B. A. J. Agric. Food Chem. 2019, 67, 11860.

[73] Roy, A.; Kundu, M.; Dhar, P.; Chakraborty, A.; Mukherjee, S.; Naskar, J.; Rarhi, C.; Barik, R.; Mondal, S. K.; Wani, M. A.; Gajbhiye, R.; Roy, K. K.; Maiti, A.; Manna, P.; Adhikari, S. ChemistrySelect 2020, 5, 4559 .

[74] Ratni, H.; Ebeling, M.; Baird, J.; Bendels, S.; Bylund, J.; Chen, K. S. ; Denk, N.; Feng, Z. H.; Green, L.; Guerard, M.; Jablonski, P.; Jacobsen, B.; Khwaja, O.; Kletzl, H.; Ko, C. P.; Kustermann, S.; Marquet, A.; Metzger, F.; Mueller, B.; Naryshkin, N. A.; Paushkin, S. V.; Pinard, E.; Poirier, A.; Reutlinger, M.; Weetall, M.; Zeller, A.; Zhao, X.; Mueller, L. J. Med. Chem. 2018, 61, 6501.

[75] Dong, Z.; Wang, Z.; Guo, Z.-Q.; Gong, S. Z.; Zhang, T.; Liu, J.; Luo, C.; Jiang, H. L.; Yang, C.-G. J. Med. Chem. 2020, 63, 4849.

[76] Marshall, A. J.; Lill, C. L.; Chao, M.; Kolekar, S. V.; Lee, W.-J.; Marshall, E. S.; Baguley, B. C.; Shepherd, P. R.; Denny, W. A.; Flanagan, J. U.; Rewcastle, G. W. Bioorg. Med. Chem. 2015, 23, 3796.

[77] Stepan, A. F.; Claffey, M. M.; Reese, M. R.; Balan, G.; Barreiro, G.; Barricklow, J.; Bohanon, M. J.; Boscoe, B. P.; Cappon, G. D.; Chenard, L. K.; Cianfrogna, J.; Chen, L. G.; Coffman, K. J.; Drozda, S. E.; Dunetz, J. R.; Ghosh, S.; Hou, X. J.; Houle, C.; Karki, K.; Lazzaro, J. T.; Mancuso, J. Y.; Marcek, J. M.; Miller, E. L.; Moen, M. A.; O'Neil, S.; Sakurada, I.; Skaddan, M.; Parikh, V.; Smith, D. L.; Trapa, P.; Tuttle, J. B.; Verhoest, P. R.; Walker, D. P.; Won, A.; Wright, A. S.; Whritenour, J.; Zasadny, K.; Zaleska, M. M.; Zhang, L.; Shaffer, C. L. J. Med. Chem. 2017, 60, 7764.

[78] Park, D.-S.; Jo, E.; Choi, J.; Lee, M.; Kim, S.; Kim, H.-Y.; Nam, J.; Ahn, S.; Hwang, J. Y.; Windisch, M. P. Eur. J. Med. Chem. 2017, $140,65$.

[79] Jadhav, S. B.; Fatema, S.; Patil, R. B.; Sangshetti, J. N.; Farooqui, M. J. Heterocycl. Chem. 2017, 54, 3299.

[80] Hussain, M.; Liu, J. H. Tetrahedron Lett. 2020, 61, 152269.

[81] Mathavan, S.; Raj, A. K. D.; Yamajala, R. B. R. D. ChemistrySelect 2019, 4, 10737.

[82] Pavithra, T.; Devi, E. S.; Nagarajan, S.; Sridharan, V.; Maheswari, C. U. Eur. J. Org. Chem. 2019, 6884.

[83] Alanine, T. A.; Galloway, W. R. J. D.; Bartlett, S.; Ciardiello, J. J.; McGuire, T. M.; Spring, D. R. Org. Biomol. Chem. 2015, 14, 1031.

[84] Chen, Z. W..; Wen, Y. L.; Ding, H.; Luo, G. T.; Ye, M.; Liu, L. X.; Xue, J. Tetrahedron Lett. 2017, 58, 13.

[85] Silpa, L.; Niepceron, A.; Laurent, F.; Brossier, F.; Penichon, M.; Enguehard-Gueiffier, C.; Abarbri, M.; Silvestre, A.; Petrignet, J. Bioorg. Med. Chem. Lett. 2016, 26, 114.

[86] Inturi, S. B.; Kalita, B.; Ahamed, A. Synth. Commun. 2018, 48, 2037.

[87] Madar, J. M.; Shastri, L. A.; Shastri, S. L.; Holiyachi, M.; Naik, N.; Kulkarni, R.; Shaikh, F.; Sungar, V. Synth. Commun. 2018, 48, 375.

[88] Sadeghzadeh, S. M.; Zhiani, R. J. Organomet. Chem. 2018, 868, 47.

[89] Alsharif, Z.; Ali, M. A.; Alkhattabi, H.; Jones, D.; Delancey, E.; Ravikumar, P. C.; Alam, M. A. New J. Chem. 2017, 41, 14862.

[90] El-Sayed, R.; Katouah, H. A. J. Heterocycl. Chem. 2019, 56, 2134.

[91] Rao, C. B.; Zhang, N.; Hu, J. N.; Wang, Y.; Liang, Y. J.; Zhang, R.; Yuan, J. W.; Dong, D. W. J. Org. Chem. 2020, 85, 4695.

[92] Sagir, H.; Rai, P.; Neha, S.; Singh, P. K.; Tiwari, S.; Siddiqui, I. R. 
RSC Adv. 2016, 6, 73924.

[93] Guchhait, S. K.; Saini, M.; Sumkaria, D.; Chaudhary, V. Chem. Commun. 2017, 53, 6941.

[94] Hoang, G. L.; Zoll, A. J.; Ellman, J. A. Org. Lett. 2019, 21, 3886.

[95] Brahmachari, G.; Karmakar, I.; Nurjamal, K. ACS Sustainable Chem. Eng. 2018, 6, 11018.

[96] Mahdavi, M.; Estabragh, R. F.; Moghimi, S.; Sayahi, M. H.; Shafiee, A.; Foroumadi, A. Synlett 2016, 27, 1359.

[97] Liu, J. D.; Zou, J. H.; Yao, J. W.; Chen, G. S. Adv. Synth. Catal. 2018, 360, 659 .

[98] Sha, X.-L.; Niu, J.-Y.; Sun, R.; Xu, Y.-J.; Ge, J.-F. Org. Chem. Front. 2017, 5, 555.

[99] Pham, P. H.; Doan, S. H.; Vuong, N. T. H; Nguyen, V. H. H.; Ha, P. T. M.; Phan, N. T. S. RSC Adv. 2018, 8, 20314.

[100] Brendel, M.; Sakhare, P. R.; Dahiya, G.; Subramanian, P.;
Kaliappan, K. P. J. Org. Chem. 2020, 85, 8102.

[101] Feng, J.-B.; Wu, X.-F. Org. Biomol. Chem. 2015, 13, 10656.

[102] Xu, T. Y.; Alper, H. Org. Lett. 2015, 17, 1569.

[103] Yuan, Y.; Wu, X.-F. Eur. J. Org. Chem. 2019, 2172.

[104] Fei, Z. A.; Zhu, Y.-P.; Liu, M.-C.; Jia, F.-C.; Wu, A.-X. Tetrahedron Lett. 2013, 54, 1222.

[105] Yang, Y.; Shu, W.-M.; Yu, S.-B.; Ni, F.; Gao, M.; Wu, A.-X. Chem. Commun. 2013, 49, 1729.

[106] Li, D.; Wang, Q.; Rao, N.; Zhang, Y.; Le, Y.; Liu, L.; Li, L. L.; Huang, L.; Yan, L. J. J. Mol. Struct. 2021, 1239, 130521.

[107] Srivastava, S.; Thakur, N.; Singh, A.; Shukla, P.; Maikhuri, V. K.; Garg, N.; Prasad, A.; Pandey, R. RSC Adv. 2019, 9, 29856.

[108] Liu, X.; Li, W.; Liu, H. Y.; Cao, H. Chin. J. Org. Chem., 2021, 41, 1759 (in Chinese).

(刘想, 李文, 刘环宇, 曹华, 有机化学, 2021, 41, 1759.)

$(\mathrm{Lu}, \mathrm{Y}$. 